\title{
Effect of low-intensity continuous training on lung function and cardiorespiratory fitness in both cigarette and hookah smokers.
}

\author{
Abdessalem Koubaa ${ }^{1,2,4}+$, Moez Triki ${ }^{3} \dagger$, Hajer Trabelsi ${ }^{4} \dagger$, Liwa Masmoudi ${ }^{4} \dagger$, \\ Khaled N. Zeghal ${ }^{1} \dagger$, Zouhair Sahnoun ${ }^{1} \dagger$, Ahmed Hakim $^{1} \dagger$
}

1. Laboratory of Pharmacology, Sfax Medicine Faculty SMF, University of Sfax, Tunisia.

2. Bizerte Sciences Faculty, Department of Biology, University of Carthage. Tunisia.

3. Laboratory of cardio-circulatory, respiratory, and hormonal adaptations to muscular exercise, Faculty of Medicine Ibn El Jazzar, University of Sousse, Tunisia.

4. Research Unit (EM2S), Higher Institute of Sport and Physical Education of Sfax, University of Sfax, Tunisia.

†All authors contributed equally to this work.

\begin{abstract}
Background: The decline in cardiorespiratory fitness and lung function was higher in smokers. Training method could mitigate some of the negative consequences of smoking among smokers unable or unwilling to quit.

Objective: To examine the effects of continuous training on lungs functional capability and cardiorespiratory fitness in smokers.
\end{abstract}

Methods: Fifteen cigarette smokers, 14 hookah smokers, and 14 nonsmokers were assigned to low-intensity continuous training (20-30 minutes of running at 40\% of maximum oxygen uptake $\left(\mathrm{VO}_{2} \mathrm{max}\right)$. Lung function and cardiorespiratory fitness parameters were determined using respectively spirometer and treadmill maximal exercise test.

Results: Continuous training improved forced expiratory volume in one second $\left(\mathrm{FEV}_{1}\right)$ and forced expiratory flow at $50 \%$ of FVC (FEF50 \%) in all participants, smokers and nonsmokers ( $\mathrm{p}<0.05)$. In contrast, forced vital capacity (FVC) improvement was significant only among cigarette smokers $(\mathrm{CS})(+1.7 \pm 2.21 \%, \mathrm{p}<0.01)$ and hookah smokers $(\mathrm{HS})(+1.3 \pm 1.7$

$\%, \mathrm{p}<0.05)$. Likewise, an improvement in cardiorespiratory fitness in both smokers groups without significant changes in diastolic blood pressure (DBP) for CS group and in velocity at maximum oxygen uptake (vVO ${ }_{2}$ max) for HS group. Conclusion: The low-intensity continuous training improves cardiorespiratory fitness and reduces lung function decline in both cigarette and hookah smokers. It seems to be beneficial in the prevention programs of hypertension. It could have important implications in prevention and treatment programs in smokers unable or unwilling to quit.

Keywords: Cigarette smokers; hookah smokers; pulmonary function; cardiorespiratory fitness; continuous training

DOI:

http: / /dx.doi.org/10.4314/ahs.v15i4.16

Cite as: Koubaa A, Triki M, Trabelsi H, Masmoudi L, Zeghal KN, Sabnoun Z, et al. Effect of low-intensity continuous training on lung function and cardiorespiratory fitness in both cigarette and bookah smokers. Afri Health Sci. 2015;15(4):1170-81. bttp:/ / dx.doi. org/10.4314/abs.v15i4.16

\section{Introduction}

The decline in fitness and lung function was significant- ly higher in smokers than in nonsmokers and could not be explained by differences in age and physical activity ${ }^{1}$.

\section{Corresponding author:}

Abdessalem Koubaa,

Laboratory of Pharmacology,

Sfax Medicine Faculty SMF,

Avenue Majida Boulila, Sfax 3029, Tunisia.

E-mail: abdessalemkoubaa@gmail.com

Tel: 0021620386968

Fax : 0021674278502
Smoking is the biggest risk factor involved in the decline of lung function. In this context, several authors have found that smoking reduces the forced expiratory volume in one second $\left(\mathrm{FEV}_{1}\right)$, forced vital capacity (FVC) and Tiffeneau index $\left(\mathrm{FEV}_{1} / \mathrm{FVC}\right)$ in both sexes24 .

The cigarettes or hookah consumption has risks of addiction, illness or death. Koubaa et al. ${ }^{5}$ assessed the harmful effects of hookah consumption compared to cigarette smokers in sedentary adult's subjects by measuring biochemical and cardiorespiratory parameters. This study reinforces the evidence that hookah consumption was associated with exposure to toxic sub- 
stances and harmful effects on cardiopulmonary function and antioxidant defense capacity and produced in some cases, the same effects as the cigarettes. Many previous studies suggest that smoking hookah has adverse effects similar to cigarettes ${ }^{6-8}$.

The findings of Saetta et al. ${ }^{9}$ indicate that cigarette smoke affects not only the airways, but also the lung parenchyma and pulmonary arteries, causing irreversible obstruction of the branches. The main risk factor for this obstruction is smoking. Thus, and according to a Swedish study that was interested in subjects aged over 76 years, nearly $66.7 \%$ of smokers presented with a chronic obstructive pulmonary disease (COPD). This data suggests that COPD is a disease that affects the majority of smokers when they live long enough ${ }^{10}$. This pathology, according to WHO statistics ${ }^{11}$, is the cause of death that will increase more in industrialized countries and will become the third leading cause of death in 2020. In Tunisia, the death rate from smoking-related COPD is $84 \%$ in men and $35 \%$ in women ${ }^{12}$.

Undoubtedly, the inhalation of cigarette or hookah smoke is associated with hypertension (HT), an in- crease in resting heart rate (HR) and at exercise and a decreased tolerance to the effort ${ }^{13}$. These effects are important firstly via the nicotine which causes an in-crease in myocardial oxygen demand and, secondly, by the functional anemia induced by the increased uptake of carbonmonoxide on the hemoglobin ${ }^{14}$. Therefore an increased tachycardia, decreased maximal oxygen consumption and harmful effect on peripheral muscle ${ }^{15,16}$ with early anaerobic threshold ${ }^{17}$. These different effects result in reduced of effort tolerance ${ }^{18,19}$.

In order to prevent and slow the progression of hypertension and improve health and cardiorespiratory performance, several previous studies have suggested that physical activity can play a central role ${ }^{20-23}$. In this context, the Canadian medical association indicated that regular moderate physical activity $(40 \%$ to $60 \%$ of $\mathrm{VO}_{2} \max$ ) for 50 to 60 minutes, 3-4 times a week was recommended in the prevention or treatment of hypertension ${ }^{24}$.Fagard et $\mathrm{al}^{22}$ confirm these results, showing a significant reduction in systolic blood pressure (SBP) and diastolic blood pressure (DBP) for a repeated exercise 3-5 times per week for 30 to $60 \mathrm{~min}$ and $40 \%$ to $50 \%$ of $\mathrm{VO}_{2}$ max. In addition, other studies using different training periods showed significant improvements in $\mathrm{VO}_{2} \mathrm{max}$ and in the rate of spontaneous walking ${ }^{20}$ and a significant decrease in fatigue, and an improvement of physical ability and life quality $^{21}$.

Exercise may have the potential to mitigate some of the negative consequences of smoking. Some studies, suggest that training at vigorous exercise intensity $(60-85 \%$ of reserve heart rate) can be a useful aid to stop smoking $^{25,26}$. To our knowledge there's lack of empirical evidence documented that such a method of physical activity has beneficial effects on physiological symptoms of smokers. Therefore, there is a need to expand the range of potentially effective harm reduction strategies among smokers unable or unwilling to quit smoking.

We would like discover a physical activity method to become a strategy so that it can improve cardiopulmonary performance and delay the lung function decline caused by smoking. It seems therefore important to assess through a co- hort study, the low-intensity continuous training effects on cardiorespiratory performance and lung function in sedentary adults, cigarette and hookah smokers.

\section{Methods \\ Participants}

A total of forty-three sedentary and healthy male smokers and non-smokers from the general community of Tunisia, which belongs to the public function (profession does not require physical exertion), volunteered to participate in this study and were recruited within pharmacology laboratory of the Faculty of Medicine, University of Sfax, Tunisia. The anthropometric characteristics of participant are shown in [Table 1]. 


\begin{tabular}{|c|c|c|c|c|}
\hline \multirow{2}{*}{ Parameters } & \multicolumn{3}{|c|}{ Means \pm SD } & \multirow{2}{*}{ ANOVA } \\
\hline & $\mathrm{NS}$ & $\mathrm{CS}$ & HS & \\
\hline Age (yrs) & $43.8 \pm 2.1$ & $43.2 \pm 2.1$ & $43.7 \pm 2.3$ & $\mathrm{p}=0.82$ \\
\hline Height $(\mathrm{cm})$ & $175.6 \pm 2.2$ & $175.9 \pm 1.5$ & $175.3 \pm 1.5$ & $\mathrm{p}=0.75$ \\
\hline Weight (kg) & $74.1 \pm 4.4$ & $74.3 \pm 2.3$ & $74 \pm 3.5$ & $\mathrm{p}=0.97$ \\
\hline BMI $\left(\mathrm{kg} \cdot \mathrm{m}^{-2}\right)$ & $24.1 \pm 1.8$ & $24 \pm 1$ & $24.1 \pm 1.2$ & $\mathrm{p}=0.99$ \\
\hline
\end{tabular}

Participants were admitted to the training program after approval by a cardiologist physician. They were normolipidemic (fasting triglycerides $<1.7 \mathrm{mmol} / \mathrm{L}$ ), nonobese. No subject used nutritional supplements or medications. Presence of any kind of disease (based on history, medical examination, and exercise stress testing), or $\mathrm{FEV} 1 / \mathrm{FVC} \%<70 \%{ }^{27,28}$, or involvement in regular physical activity or exercise program for the 12month period preceding the visit day, were also exclusion criteria. On the basis of these criteria, 9 subjects from 52 were excluded. Eventually, 43 subjects were included in subsequent tests and they were admitted to the training program.

After receiving a complete verbal description of protocol, risks and benefits of the study, subjects provided written consent to an experimental protocol approved by the Research Ethics Committee of the Faculty of Medicine, University of Sfax, Tunisia. Smokers were instructed to refrain from smoking at least one hour prior to reporting to the laboratory as suggested by Dietrich et al. ${ }^{29}$

Cigarette and hookah smokers were recruited according to the number of cigarettes and hookah per day and how long they had been smoking. We considered cigarette smokers all subjects with consumption greater or equal to 10 pack-years (PY) and an average score of tobacco dependence of $8.12 \pm 1.41$, measured by the Fagerström nicotine dependence test ${ }^{30}$. We quantified hookah consumption, as in the study of Kiter et al. ${ }^{31}$, in hookah- years (HY) and $\mathrm{kg}$ of cumulative tobacco. The tobacco used in a single hookah session weighs be tween 10 and 25 grams $^{32}$. Regular hookah smokers are those having tobacco consumption greater or equal to 5 hookah- years $(\mathrm{HY})^{33}$.
Participants were divided into three groups, and they performed a low-intensity continuous training program 3 times a week for 12 weeks. A cigarette smokers group (CS) ( $\mathrm{n}=15)$; a hookah smokers group (HS) $(\mathrm{n}=14)$ and another nonsmokers group (NS) $(\mathrm{n}=14)$. All subjects were subjected to a spirometric assessment and physical test session before and after the training program. The session includes lung function and treadmill maximal exercise test. All these measurements were performed by the same examiners to avoid methodological errors.

\section{Anthropometric measurements}

Body weight was measured to the nearest 100 grams with a calibrated electronic scale (TANITA TBF.350 model), and height was measured to the nearest $1 \mathrm{~mm}$ with a fixed stadiometer. Body mass index (BMI) was calculated with the formula: [BMI (kg.m-2) = Weight $\left.(\mathrm{kg}) / \operatorname{Height}^{2}\left(\mathrm{~m}^{2}\right)\right]$.

\section{Calculation of recovery index}

Heart rate was recorded every minute during 5 minutes after the exercise test.

Calculation of recovery index is based on two data:

Calculation of the regression index and the correlation index.

Recovery index $=$ Regression index x correlation Index

\section{Lung function assessments}

A portable spirometer (MIR Spirobank G USB Spirometer, Rome, Italy) was used to assess smokers lung function. Standard procedure requires forced vital capacity $(\mathrm{FVC})$ and forced expiratory volume in one second $\left(\mathrm{FEV}_{1}\right)$ and should be measured from a series of at least three forced expiratory curves ${ }^{34}$. This study requiredparticipants to perform three correct manoeuvres. Participants completed the spirometry assessment seated 
with a nose clip attached, the mouthpiece is placed into the mouth, lips and teeth around the mouthpiece to form a tight seal and breathe out hard and quickly until all air is expelled.

It is vital that participants inhale completely, to total lung capacity, and continue to exhale until they have fully emptied their lungs (to residual volume). Pulmonary function variables included: $\mathrm{FVC}, \mathrm{FEV}_{1}, \mathrm{FEV}_{1}, \mathrm{FVC}$ ratio, $\mathrm{FEF}_{50 \%}$ and $\mathrm{FEF}_{25-75 \%}$. Results were expressed as percentages of the predicted value to allow comparison of results across participants.

\section{Physical fitness assessment}

$V \mathrm{O}_{2}$ max and max heart rate measurements during exercise were examined through treadmill maximal exercise test (COSMED Pulmonary-Function Equipment 37 Via dei Piani di monte Savello I-00040 Rome ITALY). This dynamic and maximum test, untilfatigue, consists in increasing the speed of $1 \mathrm{kmh}^{-1}$ every $2 \mathrm{~min}$, after warm up of 5 min with a $6 \mathrm{kmh}^{-1}$ speed until the participant could no longer continue. $V \mathrm{O}_{2}$ max is reached when oxygen consumption remains at steady state despite an increase in workload. Heart rate using (Polar Electro Oy, Kempele, Finland) was monitored throughout the test and was recorded at the conclusion of every two-minute stage. The oxygen consumption $\left(\mathrm{VO}_{2}\right)$ was continually recorded and measured in real time using oxygen analyzer (Fitmate, version 1.2 PRO COSMED). At the end of the test a detailed report will be printed. Verbal encouragement was provided throughout the test to ensure that the maximal effort was achieved.

\section{Continuous training protocol}

Subjects of three groups underwent a continuous training program during a 3 -months period. Training was performed continuously for 20 minutes (first month), 25 minutes (second month) and 30 minutes (third month), three times per week at an intensity of $40 \%$ of $\mathrm{VO}_{2} \mathrm{max}$, on race track of $400 \mathrm{~m}$ at the Institute of
Sport of Sfax, Tunisia. The cones placed and spaced 20 meters on a race track. At each beep, the subject must reach the following cone. All warm-ups before training should be between $50 \%$ and $60 \%$ of maximum heart rate for a period of about 10 minutes.

It was asked of participants to run with a continuous rhythm respecting sound beeps and the requested time throughout the training session. The training load was insured by time and traveled distance and controlled by sound beeps. ( $\mathrm{T}$ : the time between two cones; d: distance between two cones; V: proposed velocity). The load increase during the training period was provided by the increase in working time and the distance covered in each session. All participants successfully completed the training period and no absences were recorded during all sessions. In addition, we have verified that there was no involvement in physical activity or exercise program throughout the 12 -week training period.

\section{Statistical analysis}

All statistical tests were processed using STATISTICA Software (StatSoft, France). The data was expressed as mean $\pm \mathrm{SD}$ (standard deviation). After normality verification with the Shapiro-Wilk's w test, and homogeneity of variances with Levene's test, parametric tests were performed. One-way ANOVA was used to indicate inter group differences in the baseline subjects' characteristics. Inter and intra-group comparisons of the variables were made by two-way ANOVA (group vs. training) with repeated measurements. Least Significant Different (LSD) post-hoc analysis was used to identify significant group differences that were indicated by one-way and two-way ANOVA. A probability level of 0.05 was selected as the criterion for statistical significance.

\section{Results}

Before and after training, we did not observe any significant difference in body-weight and BMI values between the nonsmoker and smoker groups (Table 2).

\section{Table 2. Differences in body weight and BMI values in Pres vs. Post program}

\begin{tabular}{lccccccc}
\multicolumn{3}{c}{ Parameters } & \multicolumn{3}{c}{ Before Training } & & \multicolumn{2}{c}{ After Training } & ANOVA \\
\hline & NS & CS & HS & NS & CS & HS & \\
\hline Weight $(\mathrm{kg})$ & $74.1 \pm 4,4$ & $74.3 \pm 2.3$ & $74 \pm 3.5$ & $74.3 \pm 2.9$ & $74.1 \pm 2.1$ & $74.4 \pm 1.5$ & ns \\
BMI (kg.m $\left.{ }^{-2}\right)$ & $24.1 \pm 1.8$ & $24 \pm 1$ & $24.1 \pm 1.2$ & $24 \pm 1.3$ & $24.1 \pm 0.9$ & $24.1 \pm 1$ & ns \\
\hline
\end{tabular}

$$
\mathrm{ns}=\text { not significant, } \mathrm{p}>0.05
$$


However, most of the spirometric values were higher in training program. We reported in table 3 the spirometall of non-smokers subjects and significantly different ric values in percentages of the predicted value of our to those of cigarette and hookah smokers before our entire population before training.

Table 3. Respiratory parameters of participants before training

\begin{tabular}{lcccc}
\hline \multirow{2}{*}{ Parameters } & \multicolumn{3}{c}{ Means \pm SD } & \multirow{2}{*}{ ANOVA } \\
\cline { 2 - 4 } & NS & CS & HS & \\
\hline FVC (\%) & $103.17 \pm 5.3$ & $93.2 \pm 6.4^{* * *}$ & $94.6 \pm 5.2^{* *}$ & $\mathrm{p}<0,001$ \\
FEV1 (\%) & $102.08 \pm 4.6$ & $99.8 \pm 4.2$ & $94.7 \pm 5.9^{* * \#}$ & $\mathrm{p}=0,005$ \\
PEF (\%) & $109.58 \pm 4.4$ & $102.9 \pm 4.6^{* *}$ & $100.9 \pm 5.7^{* * *}$ & $\mathrm{p}<0,001$ \\
FEV1/FVC (\%) & $99.12 \pm 5.6$ & $107.45 \pm 7.5^{*}$ & $100.45 \pm 9.2 \#$ & $\mathrm{p}=0,035$ \\
FEF $25-75 \%$ & $101.92 \pm 7.1$ & $92.8 \pm 5.4^{* * *}$ & $90.8 \pm 3.3^{* * *}$ & $\mathrm{p}<0,001$ \\
$(\%)$ FEF $_{50 \%} \%$ & $98.08 \pm 6.1$ & $87.9 \pm 4.8^{*}$ & $86 \pm 6.3^{* * \#}$ & $\mathrm{p}=0,005$
\end{tabular}

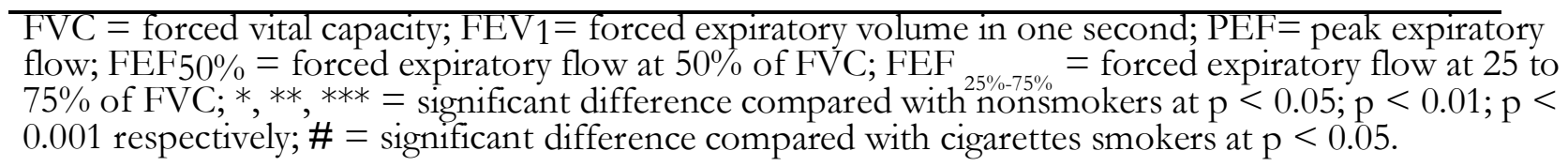

Compared to nonsmokers group, ANOVA showed sig- Furthermore, the FEV 1 of CS group tends to be lower nificant differences for all measured parameters. For than of NS group, but the difference was not signifiexplored values of FVC, PEF and FEF 25-75\%, statis- cant. The HS group also showed a low level of FEF tical analysis showed no difference among cigarette and 50\% compared to the two groups CS $(\mathrm{P}<0.05)$ and hookah smokers. NS $(\mathrm{P}<0.01)$.

Application of LSD post-hoc test showed a similar Training effect on lung function significant difference $(\mathrm{p}<0.05)$ in $\mathrm{FEV}_{1}, \mathrm{FEV}_{1} / \mathrm{FVC}$, The improvement rate in the respiratory functional and FEF $50 \%$ of CS subjects compared to HS subjects. exploration results after the training period, is summarized in table 4. 
Table 4. Improvement rate $(\Delta)$ of respiratory parameters of the three groups (NS, CS, HS).

\begin{tabular}{lcccc}
\hline Parameters & \multicolumn{3}{c}{ Means \pm SD } & \multirow{2}{*}{ ANOVA } \\
\cline { 2 - 4 } & NS & CS & HS & \\
\hline FVC (\%) & $103.17 \pm 5.3$ & $93.2 \pm 6.4^{* * *}$ & $94.6 \pm 5.2^{* *}$ & $\mathrm{p}<0,001$ \\
FEV1 (\%) & $102.08 \pm 4.6$ & $99.8 \pm 4.2$ & $94.7 \pm 5.9^{* * \#}$ & $\mathrm{p}=0,005$ \\
PEF (\%) & $109.58 \pm 4.4$ & $102.9 \pm 4.6^{* *}$ & $100.9 \pm 5.7^{* * *}$ & $\mathrm{p}<0,001$ \\
FEV1/FVC (\%) & $99.12 \pm 5.6$ & $107.45 \pm 7.5^{*}$ & $100.45 \pm 9.2 \#$ & $\mathrm{p}=0,035$ \\
FEF25-75\% $\%)$ & $101.92 \pm 7.1$ & $92.8 \pm 5.4^{* * *}$ & $90.8 \pm 3.3^{* * *}$ & $\mathrm{p}<0,001$ \\
FEF50\% $\%$ (\%) & $98.08 \pm 6.1$ & $87.9 \pm 4.8^{*}$ & $86 \pm 6.3^{* * \#}$ & $\mathrm{p}=0,005$ \\
\hline
\end{tabular}

$\mathrm{FVC}=$ forced vital capacity; FEV1 $=$ forced expiratory volume in one second; $\mathrm{PEF}=$ peak expiratory flow; FEF50\% = forced expiratory flow at 50\% of FVC; FEF 25\%-75\% = forced expiratory flow at 25 to $75 \%$ of FVC; ns = not significant; $\dagger, \boldsymbol{H}=$ significant difference in Pre vs. Post program at $\mathrm{p}<$ $0.05 ; \mathrm{p}<0.01$, respectively.

The three-month continuous training period, induces changes in respiratory parameters, however, they vary according to the group. This change did not show significant differences in PEF, FEV $1 /$ FVC and FEF 25$75 \%$ measured after the training period.

The training period produces an increase in FVC of all our participants; however, this improvement was significant only among smokers. It is of the order of +1.7 $\pm 2.21 \%(\mathrm{p}<0.01)$ for CS group and $+1.3 \pm 1.7 \%(\mathrm{p}$ $<0.05)$ for HS group. In addition, all our subjects ben- efited a significant increase in $\mathrm{FEV}_{1}$ after the training program (Table 3). Thus, the improvement was $+1.83 \pm$ $2.69 \%$ of NS group $(p<0.05),+1.9 \pm 2.13 \%(p<0.05)$ in CS group and $+1.7 \pm 2 \%(\mathrm{p}<0.05)$ for the HS group. The FEF $50 \%$ of the three groups NS, CS and HS follows the same trend as the $\mathrm{FEV}_{1}$, with significant differences $(p<0.05)$, representing increases of $+1.08 \pm$ $2.19 \%,+1 \pm 2.36 \%$ and $+1.6 \pm 2.5 \%$, respectively.

\section{Training effect on cardiorespiratory fitness}

The results of maximal exercise test of the three groups before training period are summarized in table 5 . 
Table 5. Cardiorespiratory parameters of all participants before training

\begin{tabular}{|c|c|c|c|c|}
\hline \multirow{2}{*}{ Parameters } & \multicolumn{3}{|c|}{ Means \pm SD } & \multirow{2}{*}{ - ANOVA } \\
\hline & NS & CS & HS & \\
\hline Resting HR (bpm) & $82,08 \pm 4,6$ & $88,8 \pm 4,2^{* * *}$ & $90,7 \pm 3 * * *$ & $\mathrm{p}<0,001$ \\
\hline $\mathrm{SBP}(\mathrm{mm} \mathrm{Hg})$ & $124,33 \pm 7,1$ & $140,2 \pm 3,1^{* * *}$ & $143,2 \pm 4,7^{* * *}$ & $\mathrm{p}<0,001$ \\
\hline $\mathrm{DBP}(\mathrm{mm} \mathrm{Hg})$ & $84,25 \pm 6,8$ & $91,1 \pm 2,4^{* *}$ & $93,4 \pm 3,4^{* * *}$ & $\mathrm{p}<0,001$ \\
\hline $\mathrm{vVO}, \max \left(\mathrm{km} \cdot \mathrm{h}^{-1}\right)$ & $11,18 \pm 0,2$ & $10,26 \pm 0,2 * * *$ & 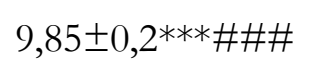 & $\mathrm{p}<0,001$ \\
\hline $\mathrm{VO}_{2} \max \left(\mathrm{ml} \cdot \mathrm{min}^{-1} \cdot \mathrm{kg}^{-}\right.$ & $39 \pm 0,7$ & $35,78 \pm 0,9 * * *$ & $34,35 \pm 0,8 * * * \# \# \#$ & $\mathrm{p}<0,001$ \\
\hline Recovery index & $17,9 \pm 0,8$ & $17,31 \pm 1$ & $15,99 \pm 1 * * * \# \#$ & $\mathrm{p}<0,001$ \\
\hline
\end{tabular}

$\mathrm{HR}=$ heart rate; $\mathrm{bpm}=$ beats per minute; $\mathrm{SBP}=$ systolic blood pressure; $\mathrm{DBP}=$ diastolic blood pressure; $\mathrm{vVO}_{2} \max =$ velocity at maximum oxygen uptake; $\mathrm{VO} 2 \mathrm{max}=$ maximum oxygen uptake; $* *, * * *=$ significant differences compared to nonsmokers at $\mathrm{p}<0.01, \mathrm{p}<0.001$ respectively; \#\#, \#\#\# = significant differences compared to CS at $\mathrm{p}<0.01 ; \mathrm{p}<0.001$, respectively.

The (LSD) post-hoc test showed that the two groups CS and HS had resting HR, SBP and DBP similar and significantly higher than those of nonsmokers $(\mathrm{p}<$ 0.001). Similarly, no significant difference in these values was revealed between the two smoker groups.

Regarding the $\mathrm{VO}_{2}$ max, $\mathrm{v} V \mathrm{O}_{2}$ max and recovery index, the statistical analysis showed significant differences between the two smoking groups $(\mathrm{p}<0.001, \mathrm{p}<0.001$ and $\mathrm{p}<0.01$, respectively). Similarly, we have registered in the values of $\mathrm{vVO}_{2}$ max and $\mathrm{VO}_{2}$ max, significant differences between smoker and nonsmoker groups ( $\mathrm{p}$ $<0.001)$. The recovery index was better in nonsmokers compared to cigarette smokers $(\mathrm{p}<0.001)$ and in cigarette smokers versus hookah smokers $(\mathrm{p}<0.01)$.

After the continuous training period, participants showed different improvements (Table 6). Significant changes in resting HR for the three groups NS, CS and HS were observed after training, with declines of -1.75 $\pm 2 \mathrm{bpm}(\mathrm{P}<0.05), 2.5 \pm 34 \mathrm{bpm}(\mathrm{P}<0.01)$ and -2.2 $\pm 3.1 \mathrm{bpm}(\mathrm{P}<0.05)$, respectively. Similarly, there was a decrease of SBP for both smoker groups $(\mathrm{P}<0.05)$. In contrast, the decrease in DBP was significant only for the HS group $(\mathrm{P}<0.01)$, by a decrease of $-2.4 \pm 3.4(\mathrm{~mm}$ $\mathrm{Hg})$.

The low-intensity continuous training induced also significant increases of $\mathrm{vVO}_{2}$ max for subjects of $\mathrm{NS}$ and $\mathrm{CS}$ groups and $\mathrm{VO}_{2}$ max for the subjects of the CS and HS groups. Finally, the recovery index results showed most improved recoveries for the subjects of the three groups (NS: $+0.44 \pm 0.4$; CS: $+0.47 \pm 0.6$; HS: $+098 \pm 0.8)$. 
Table 6. Improvement rate $(\Delta)$ of cardiorespiratory values in Pre vs. Post training program

\begin{tabular}{|c|c|c|c|c|}
\hline \multirow{2}{*}{ Parameters } & \multicolumn{3}{|c|}{ Means $\pm \mathrm{SD}$} & \multirow{2}{*}{ ANOVA } \\
\hline & NS & CS & HS & \\
\hline Resting HR (bpm) & $82,08 \pm 4,6$ & $88,8 \pm 4,2^{* * *}$ & $90,7 \pm 3 * * *$ & $\mathrm{p}<0,001$ \\
\hline $\mathrm{SBP}(\mathrm{mm} \mathrm{Hg})$ & $124,33 \pm 7,1$ & $140,2 \pm 3,1 * * *$ & $143,2 \pm 4,7 * * *$ & $\mathrm{p}<0,001$ \\
\hline $\mathrm{DBP}(\mathrm{mm} \mathrm{Hg})$ & $84,25 \pm 6,8$ & $91,1 \pm 2,4^{* *}$ & $93,4 \pm 3,4 * * *$ & $\mathrm{p}<0,001$ \\
\hline $\mathrm{vVO}_{2} \max \quad\left(\mathrm{km} \cdot \mathrm{h}^{-1}\right)$ & $11,18 \pm 0,2$ & $10,26 \pm 0,2^{* * *}$ & $9,85 \pm 0,2 * * * \# \# \#$ & $\mathrm{p}<0,001$ \\
\hline $\mathrm{VO}_{2} \max \left(\mathrm{ml} \cdot \mathrm{min}^{-1} \cdot \mathrm{kg}^{-1}\right)$ & $39 \pm 0,7$ & $35,78 \pm 0,9 * * *$ & $34,35 \pm 0,8 * * * \# \# \#$ & $\mathrm{p}<0,001$ \\
\hline Recovery index & $17,9 \pm 0,8$ & $17,31 \pm 1$ & $15,99 \pm 1 * * * \# \#$ & $\mathrm{p}<0,001$ \\
\hline
\end{tabular}

\section{Discussion}

Sedentary lifestyle, decline of lung function and low cardiorespiratory capacity are recognized as the main predictors of morbidity 35 and mortality 36,37 . Indeed, several studies have examined, using different protocols in different cases, the effect of exercise training on aerobic capacity and lung function. However, to our knowledge, no study has determined the effect of a continuous training program on these capacities among male adults unable or refuse to quit smoking. In fact, the aim of our study was to determine the contribution of 12 -week low-intensity continuous training on lung function performance and aerobic fitness in cigarette and hookah smokers. Data of this study show the relationship between physical activity, cardiorespiratory capacity and lung function in healthy male, smokers and non-smokers.
The low-intensity continuous training was strongly associated with better values of the treadmill maximal exercise test. This finding was consistent with other studies $^{38,39}$. This study revealed considerable changes in $\mathrm{VO}_{2} \mathrm{max}$ and recovery index of all smoker participants. However, in the HS group, we found a greater improvement of two recorded values (see Fig. 1). In this context, Daussin et al. ${ }^{40}$ showed a significant increase in $\mathrm{VO}_{2}$ max of the subjects who participated in a continuous training program for 8 weeks. Our findings support the results of MacDougall et al. ${ }^{38}$, Harmer et al. ${ }^{39}$, Macfarlane et al. ${ }^{41}$, Tijonna et al. ${ }^{42}$ and Daussin et al. ${ }^{40}$ who reported significant increases in $\mathrm{VO}_{2} \mathrm{max}$ values after various training programs. In contrast, a related study that was conducted by Mazoochi et al. 43 showed no continuous training effect on $\mathrm{VO}_{2}$ max. The results of this study can be confirmed by those of Denis et al. ${ }^{44}$.

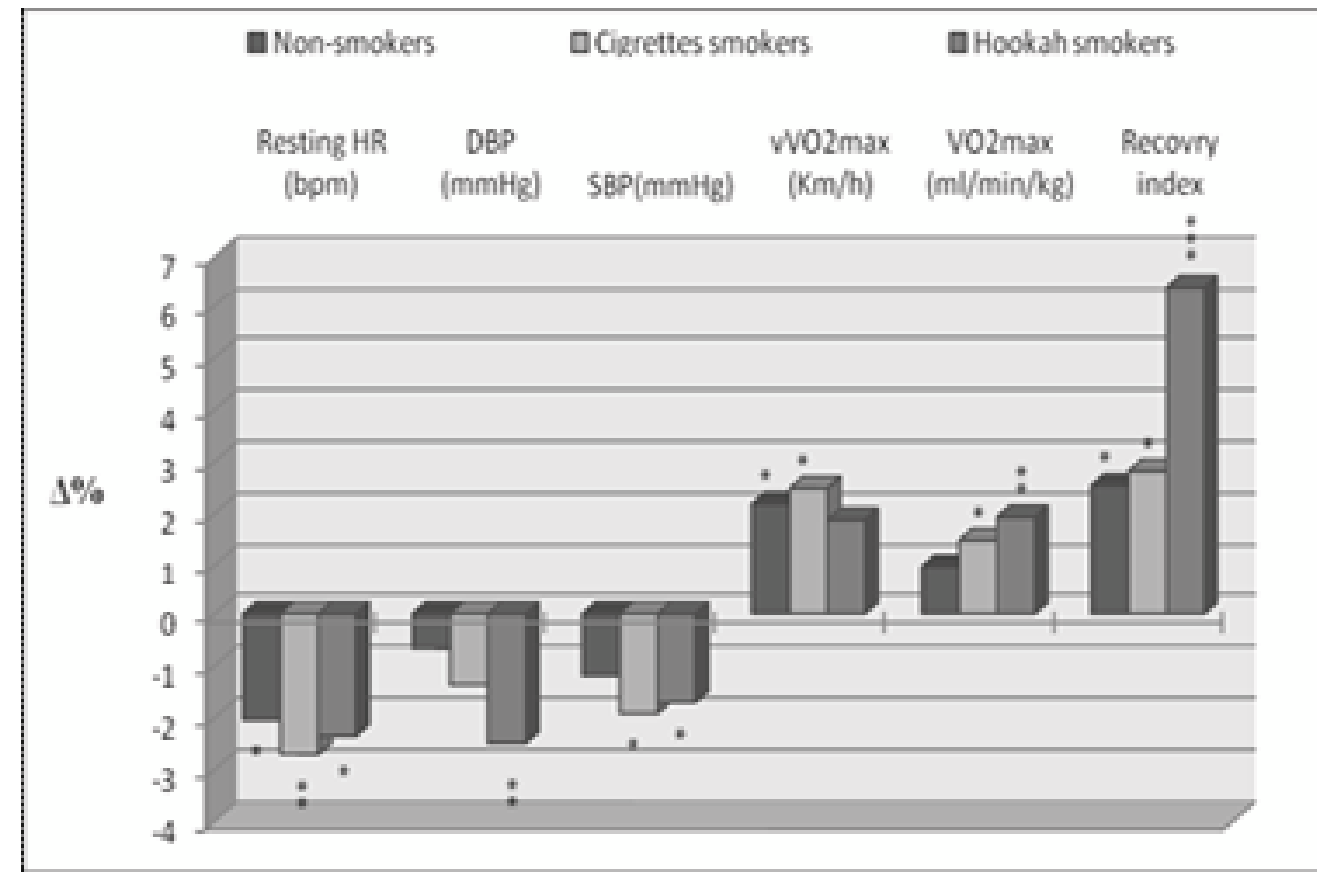

Fig 1. Improvement rate in percentage $(\Delta \%)$ of cardiorespirory parameters in Pre vs. Post program 
On the other hand, low-intensity continuous training induced a significant decrease in blood pressure and resting HR. The result is a significant reduction in SBP of $-2 \%$ for CS group and $-1.8 \%$ for the HS group, and only significant decrease of $-2.5 \%$ of DBP in HS group (Fig.1). These favorable changes resulting from the continuous training on this two recorded values are in agreement with studies of Lawal \& Kankanala 45 and
Laterza et al. ${ }^{46}$ and different from conclusions of Ferrier et al. ${ }^{47}$. Similar results to our findings were reported by Westhoff et $\mathrm{al}^{48}$. Their findings show a significant decrease in SBP and DBP of $-8.5 \pm 8.2 \mathrm{~mm} \mathrm{Hg}$ and $-5.1 \pm 3.7 \mathrm{~mm} \mathrm{Hg}$, respectively. This partial difference in results may be explained in part by the implemented protocols diversity (Training methods, protocol duration, participants' age, smoking habits etc.) and the individual responses of each participant to exercise.

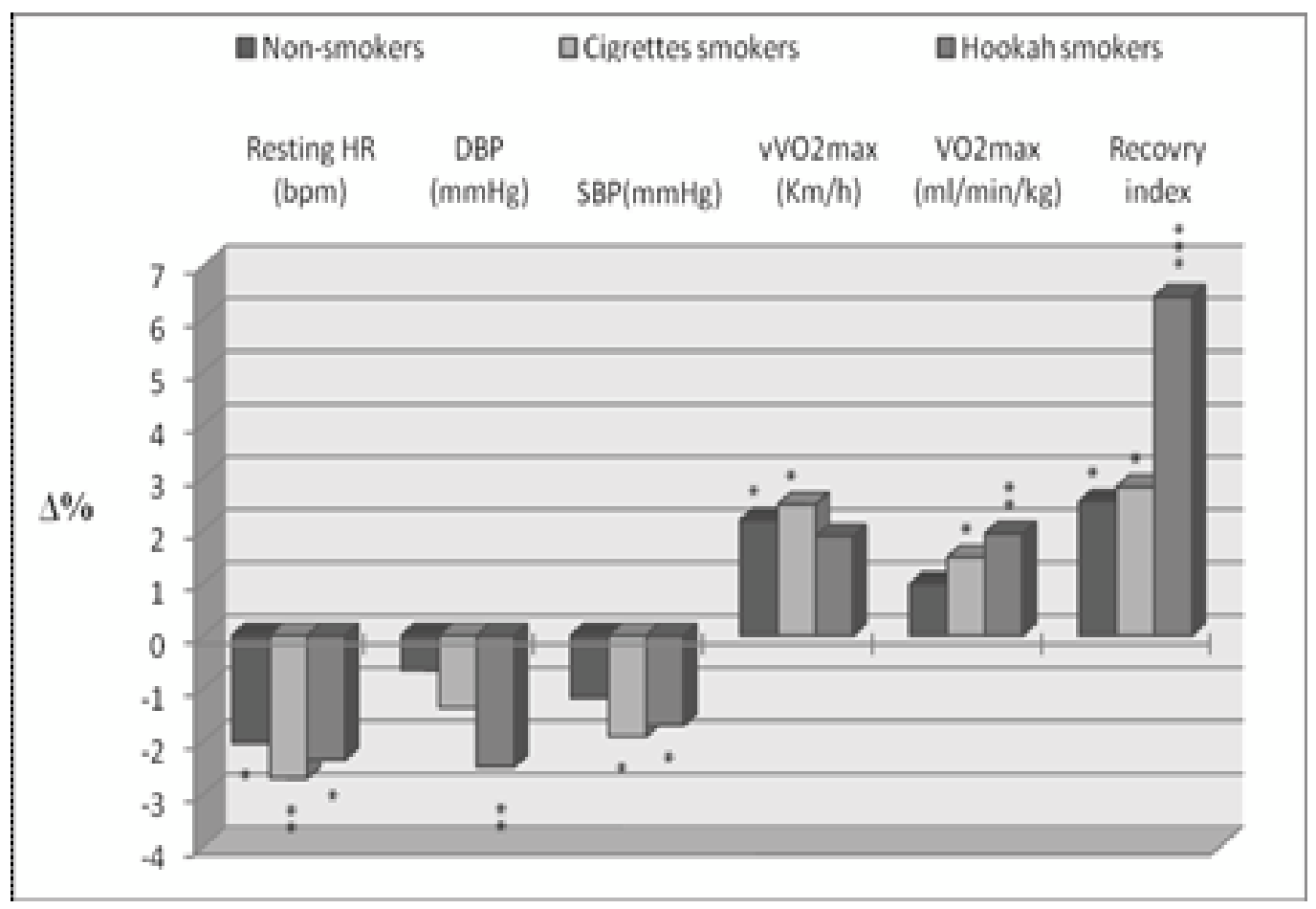

Fig 2. Improvement rate in percentage $(\Delta \%)$ of lung function parameters in Pre vs. Post program

Exercise is an important component of pulmonary rehabilitation and may be associated with physiological and psychological benefits ${ }^{49}$. Although the respiratory rehabilitation programs improve the quality of life and some physiological measures, the improvements in $\mathrm{FEV}_{1}$ levels were not reported consistently ${ }^{2}$. In our study, all participants, smokers and nonsmokers had higher levels of $\mathrm{FEV}_{1}$ and FVC after this continuous training program. The improvement was about $+2 \%$ and from $+0.9 \%$ to $+2 \%$, respectively (Fig. 2 ). Our results confirm the findings of Mehrotra et al. ${ }^{50}$, who reported that lung function was better in most active subjects than sedentary subjects. However, there was no significant difference of $\mathrm{FEV}_{1} / \mathrm{FVC}$ in Pre vs. Post program. This is explained by the pulmonary efficiency weakness of our participants. These results are consistent with the findings of Cheng et al. ${ }^{51}$.

The cigarette smoker participants who had the lowest FVC before training protocol, tended to have the best improvement among the three groups after training $(\approx$ $+2 \%$ ). This may suggest that the respiratory system response to physical activity among CS group is higher than in HS or NS groups.

In summary, our analysis suggests that a low-intensity continuous training program was associated with an improved cardiorespiratory fitness and aspect of physiological wellness. This improvement was more marked in smokers than in nonsmokers, but the respiratory func- 
tion change contributed little to this association for all participants after 12 -weeks training.

\section{Conclusion}

The present study demonstrates that low-intensity continuous training improves cardiorespiratory fitness. Intensity and training volume have been closely monitored to demonstrate the continuous exercise importance in reducing lung function decline in cigarette and hookah smokers. Likewise, physical training with continuous exercises seems to be beneficial in hypertension prevention. Finally, these results could have important implications in prevention and treatment programs in both cigarette and hookah smokers unable or unwilling to quit.

\section{Practical implications}

- Smokers before training have a reduced lung function and worst cardiorespiratory fitness compared with no smokers.

- Significant improvements in FEV 1 and FEF50 \% among smokers and nonsmokers after training.

- Significant improvements in FVC only in smokers

- Improvement in cardiorespiratory capacity is significantly higher in smokers than in nonsmokers.

- Smokers unable to quit smoking could focus at practicing leisure time physical activity regularly to reduce the decline of lung function and cardiorespiratory capacity.

\section{Limitations of the study}

The lack of a control group may be considered a limitation of the present study (smokers group follow the same daily activity during the same training period). I also think that future research should include a group of passive smokers. Likewise, the relatively small sample size could have limited our ability to detect group differences in the chosen parameters. This is indeed a limitation of this work, and should be considered relative to our findings.

\section{Conflict of interest:}

The authors declare that there is no conflict of interest regarding the publication of this article.

Source(s) of support: This research was supported by a grant from the laboratory of Pharmacology, Faculty of Medicine of Sfax, Tunisia.

\section{Acknowledgement}

The authors wish to express their sincere gratitude to all the participants who took part in this study for their maximal effort and cooperation. This study was conducted with the approval of the Research Ethics Committee of the faculty of medicine of Sfax, Tunisia.

\section{References}

1. Sandvik L, Erikssen G, Thaulow E. Long term effects of smoking on physical fitness and lung function: a longitudinal study of 1393 middle aged Norwegian men for seven years. BMJ. 1995; 311: 715-PubMed ;8.

2. Kerstjens HA, Rijcken B, Schouten JP, Postma DS. Decline of FEV1 by age and smoking status: facts, figures, and fallacies. Thorax. 1997; 52: 820-PubMed ;7.

3. Belousova EG, Haby MM, Xuan W, et al. Factors that affect normal lung function in white Australian adults. Chest. 1997; 112: 1539- PubMed ;46.

4. Twisk JW, Staal BJ, Brinkman MN, Kemper HC, van Mechelen W. Tracking of lung function parameters and the longitudinal relationship with lifestyle. Eur Respir J. 1998; 12: 627-PubMed ;34.

5. Koubaa A, Trabelsi H, Masmoudi L, Triki M, Sahnoun Z, Zeghal K, et al. Water pipe tobacco smoking and cigarette smoking: comparative analysis of the smoking effects on antioxidant status, lipid profile and cardiopulmonary quality in sedentary smokers Tunisian. IJPSI. 2013; 2: 51-7 PubMed .

6. Neergaard J, Singh P, Job J, Montgomery S. Waterpipe smoking and nicotine exposure: A review of the current evidence. Nicotine \& Tobacco Research. 2007;

9: 987-PubMed ;94.

7. Maziak W, Rastam S, Ibrahim I, Ward KD, Shihadeh A, Eissenberg T. CO exposure, puff topography, and subjective effects in waterpipe tobacco smokers. Nicotine \& Tobacco Research. 2009; 11: 806-11.

8. Salameh P, Bacha ZA, Waked M. Saliva cotinine and exhaled carbon monoxide in real life waterpipe smokers: A post hoc analysis. Tobacco Use Insights. 2009; 2: $1-10$.

9. Saetta M, Turato G, Maestrelli P, Mapp CE, Fabbri LM. Cellular and structural bases of chronic obstructive pulmonary disease. Am J Respir Crit Care Med. 2001; 163: 1304-9.

10. Lundback B, Lindberg A, Lindstrom M, Ronmark E, Jonsson AC, Jonsson E, et al. Not 15 but $50 \%$ of smokers develop COPD?--Report from the Obstructive Lung Disease in Northern Sweden Studies. Respir Med. 2003; 97: 115-22 PubMed .

11. World Health Organization: The World health re- 
port on the global tobacco epidemic. The mpower package; 2008 [downloaded 2012 January 25]. Available from: http://www.who.int/tobacco/mpower/mpower_report_full_2008.pdf

12. Fakhfakh R, Hsairi M, Ben Romdhane H, Achour

N. Mortality due to smoking in Tunisia in 1997. Tunis Med. 2001; 79: 408-12.

13. McDonough P, Moffatt RJ. Smoking-induced elevations in blood carboxyhaemoglobin levels. Sports Med. 1999; 27: 275-83 PubMed .

14. Butts NK, Golding LA. Effect of 24 hours of smoking withdrawal on cardiorespiratory functions at rest and exercise. J Sports Med Phys Fitness. 1979; 19: 38996.

15. Petersen AM, Magkos F, Atherton P, Selby A, Smith $\mathrm{K}$, Rennie MJ, et al. Smoking impairs muscle protein synthesis and increases the expression of myostatin and MAFbx in muscle. Am J Physiol Endocrinol Metab. 2007;

293: e843-48.

16. Montes OM, Loeb E, Torres SH, De SJ, Hernandez N, Talamo C. Peripheral muscle alterations in nonCOPD smokers. Chest. 2008; 133: 13-8 PubMed .

17. Hirsch GL, Sue DY, Wasserman K, Robinson TE, Hansen JE. Immediate effects of cigarette smoking on cardiorespiratory responses to exercise. J Appl Physiol. 1985; 58: 1975-81.

18. Sidney S, Sternfeld B, Gidding SS, Jacobs DR Jr, Bild DE, Oberman A, et al. Cigarette smoking and submaximal exercise test duration in a biracial population of young adults: the CARDIA study. Med Sci Sports Ex-erc. 1993; 25: 911-6.

19. Song EY, Lim CL, Lim MK. A comparison of maximum oxygen consumption, aerobic performance, and endurance in young and active male smokers and nonsmokers. Mil Med. 1998; 163: 770-4 PubMed .

20. BenekeR, Meyer K. Walking performance and economy in chronic heart failure patients pre and post exercise training. EurJ Appl Occup Pbysiol. 1997; 75: 246-51.

21. Oka RK, De Marco T, Haskell WL, Botvinick E. Impact of a home-based walking and resistance training program on quality of life in patients with heart failure. Am J Cardiol. 2000; 85: 365-9 PubMed .

22. Fagard RH. Exercise characteristics and the blood pressure response to dynamic physical training. Med Sci Sports Exerr. 2001; 33: S484-92.

23. Silva MS, Bocchi EA, Guimaraes GV, Padovani CR. Benefits of exercise training in the treatment of heart failure: study with a control group. Arq Bras Cardiol. 2002; 79: 351-62 PubMed.

24. Campbell NR, Burgess E, Taylor G, Wilson E,
Cléroux J, Fodor JG, et al. Lifestyle changes to prevent and control hypertension: do they work? A summary of the Canadian consensus conference. CMAJ. 1999; 160: 1341-3 PubMed .

25. Bock MC, Marcus BH, King TK, Borrelli B, Roberts MR. Exercise effects on withdrawal and mood among women attempting smoking cessation. Addictive Behaviors; 1999; 399-410.

26. Marcus BH, Lewis BA, Hogan J, King TK, AlbrechtAE, Bock B, et al. The efficacy of moderate-intensity exercise as an aid for smoking cessation in women: a randomized controlled trial. Nicotine Tob Res. 2005; 7: 871-80.

27. Lenfant C, Khaltaev N, editors. Global initiative for Chronic Obstructive Lung Disease, Global strategy for the diagnosis, management, and prevention of chronic obstructive pulmonary disease NHLBI/WHO workshop report, 2701. Updated 2005.

http://www.g oldcopd.org/uploads/user s/files /

GOLDWkshp05Clean.pdf

28. Chronic obstructive pulmonary disease: Management of chronic obstructive pulmonary disease in adults in primary and secondary care (partial update); 2010. http:/ / www.nice.org.uk/guidance/cg101/resources / guidance-chronic-obstructive-pulmonary-disease-pdf.

29. Dietrich M, Block G, Norkus E.P, Hudes M, Trabe M.G, Cross L. Smoking and exposure to environmental tobacco smoke decrease some plasma antioxidants and increase gamma-tocopherol in vivo after adjustment for dietary antioxidant intakes, Am J Clin Nutr, vol. 2003;

77: 160-166.

30. Heatherton TF, Kozlowski LT, Frecker RC, Fagerström KO. The Fagerström Test for Nicotine Dependence: a revision of the Fagerström Tolerance Questionnaire. BrJ Addict. 1991; 86: 1119-27 PubMed .

31. Kiter G, Ucan ES, Ceylan E, Kilinc O. Water-pipe smoking and pulmonary functions. Respir Med. 2000; 94: 891-4 PubMed .

32. Knishkowy B, Amitai Y. Water-pipe (narghile) smoking: an emerging health risk behavior. Pediatrics. 2005; 116: E113-E9.

33. Ben Saad H. The narghile and its effects on health. Part I: The narghile, general description and properties. Rev Pneumol Clin. 2009; 65: 369-75 PubMed .

34. Miller MR, Hankinson J, Brusasco V, Burgos F, Casaburi R, Coates A, et al. Standardisation of spirometry. EurRespir J. 2005; 26: 319-38 PubMed .

35. Dunn AL, Marcus BH, Kampert JB, Garcia ME, Kohl HW, Blair SN. Comparison of lifestyle and structured interventions to increase physical activity and car- 
diorespiratory fitness: a randomized trial. JAMA. 1999; 281: 327-PubMed ;34.

36. Neas LM, Schwartz J. Pulmonary function levels as predictors of mortality in a national sample of US adults. Am J Epidemiol. 1998; 147: 1011- PubMed ;18.

37. Schunemann HJ, Dorn J, Grant BJ, Winkelstein W $\mathrm{Jr}$, Trevisan M. Pulmonary function is a long-term predictor of mortality in the general population: 29-year follow-up of the Buffalo Health Study. Chest. 2000; 118:656-PubMed ;64.

38. MacDougall JD, Hicks AL, MacDonald JR, McKelvie RS, Green HJ, et al. Muscle performance and enzymatic adaptations to sprint interval training. I Appl Physiol. 1998; 84: 2138-42.

39. Harmer AR, McKenna MJ, Sutton JR, Snow RJ, Ruell PA, Booth J, et al. Skeletal muscle metabolic and ionic adaptations during intense exercise following sprint training in humans. J Appl Physiol. 2000; 89: 1793-

803 PubMed .

40. Daussin FN, Zoll J, Dufour SP, Ponsot E, Lonsdorfer-Wolf E, Doutreleau S, et al. Effect of interval versus continuous training on cardiorespiratory and mitochondrial functions; relationship to aerobic performance improvements in sedentary subjects. Am J Physiol Regul Integr Comp Physiol. 2008. 295: R264-72.

41. Macfarlane DJ, Taylor LH, Cuddihy TF. Very short intermittent vs continuous bouts of activity in sedentary adults. Preventive Medicine. 2006; 43: 332-6.

42. Tjønna AE, Lee SJ, Rognmo Ø, Stølen TO, Bye A, Haram PM et al. Aerobic interval training versus continuous moderate exercise as a treatment for the metabolic syndrome: a pilot study. Circulation. 2008; 118: 346-54 PubMed.

43. Mazoochi M, Fateminezhad SE, Mazoochi T. Ef- fects of Continuous and Interval Training on Different Fitness Parameters in Athletes. World Applied Sciences Journal. 2013; 28: 312-5.

44. Denis C, Fouquet R, Poty P, Geyssant A, Lacour JR. Effect of 40 weeks of endurance training on the anaerobic threshold. Int J Sports Med. 1982; 3: 208-14

PubMed .

45. Wahab B Lawal, Venkatseswarul Kankanala. Post- er: Effects of intermittent and continuous training on the blood pressure of Nigerian adolescents and chil- dren. Br J Sports Med. 2010; Br J Sports Med 2010;44:128 doi:10.1136/bjsm.2010.078725.94

46. Laterza MC, de Matos LD, Trombetta IC, Braga AM, Roveda F, Alves MJ, et al. Exercise training restores baroreflex sensitivity in never trained hypertensive patients. Hypertension. 2007; 49: 1298-306.

47. Ferrier KE, Waddell TK, Gatzka CD, Cameron JD, Dart AM, Kingwell BA. Aerobic exercise training does not modify large-artery compliance in isolated systolic hypertension. Hypertension. 2001; 38: 222- PubMed ;6.

48. Westhoff TH, Franke N, Schmidt S, Vallbracht-Israng $\mathrm{K}$, Meissner $\mathrm{R}$, Yildirim $\mathrm{H}$, et al. Too old to benefit from sports? The cardiovascular effects of exercise training in elderly subjects treated for isolated systolic hypertension. Kidney Blood Press Res. 2007; 30: 240-7.

49. Ries AL. The importance of exercise in pulmonary rehabilitation. Clin Chest Med. 1994; 15: 327-37.

50. Mehrotra PK, Varma N, Tiwari S, Kumar P. Pulmonary functions in Indian sportsmen playing different sports. Indian J Physiol Pharmacol. 1998; 42: 412- PubMed;6.

51. Cheng YJ, Macera CA, Addy CL, Sy FS, Wieland D, Blair SN. Effects of physical activity on exercise tests and respiratory function. BrJ Sports Med. 2003; 37: 5218 PubMed . 